\title{
O uso da acupuntura no tratamento de lúpus eritematoso sistêmico: relato de caso
}

\author{
The use of acupuncture in the treatment of Systemic Lupus Erythematosus: \\ case report \\ El uso de la acupuntura en el tratamiento del Lupus Eritematoso Sistémico: \\ reporte de caso
}

\author{
Magda Fardim DALCIN \\ Fagner Cardoso ALVES \\ Jeferson de Oliveira SALVI
}

Departamento de Farmácia, Centro Universitário Luterano de Ji-Paraná, CEULJI/ULBRA,

Av. Engenheiro Manfredo Barata Almeida da Fonseca, 76907-438 Ji-Paraná-RO, Brasil

\begin{abstract}
Resumo
O Lúpus Eritematoso Sistêmico (LES) é uma doença autoimune de origem multifatorial caracterizada pela produção de auto anticorpos patogênicos. Segundo a Medicina Tradicional Chinesa (MTC) as patologias podem ser desencadeadas por uma desarmonia energética do organismo, considerando a saúde como resultado do equilíbrio da energia ( $Q i)$ o qual pode ser alcançado por meio da acupuntura. Este artigo relata o caso de uma paciente de 19 anos submetida a 26 sessões de tratamento com acupuntura por meio da auriculoterapia e associação sistêmica, buscando a diminuição dos sinais e sintomas relacionados ao LES. Observou-se redução em torno de $75 \%$ nos domínios da dor e da ansiedade, assim como, na diminuição da dose do corticoide e do Índice de Massa Corporal (IMC). Desta forma, a acupuntura demonstrou-se efetiva como tratamento da dor, na diminuição da ansiedade e como opção não farmacológica para a corticoterapia com inversão dos sintomas da síndrome de Cushing, redução do peso e do IMC, além dos sinais e sintomas relacionados ao Lúpus Eritematosos Sistêmico. Sugere-se a continuidade da investigação, considerando um número maior de pacientes, para que se evidencie a reprodutibilidade dos procedimentos adotados e se estabeleça um protocolo clínico seguro.
\end{abstract}

Descritores: Acupuntura; Lúpus Eritematoso Sistêmico; Auriculoterapia; Corticosteroides.

\begin{abstract}
Systemic lupus erythematosus (SLE) is an autoimmune disease of multifactorial origin characterized by the production of pathogenic autoantibodies. According to Traditional Chinese Medicine (TCM) the conditions may be triggered by an energy disharmony of the body, considering health as a result of the energy balance $(Q i)$ as can be achieved through acupuncture. This article reports the case of a patient of 19 years underwent 26 treatment sessions with acupuncture through auricular and systemic associations seeking the reduction of signs and symptoms related to SLE. It was observed a reduction of about $75 \%$ in the areas of pain and anxiety, as well as the decrease in the dose of corticosteroids and Body Mass Index (BMI). Thus, acupuncture shown to be effective as a treatment for pain, decreased anxiety and as non-pharmacological option for steroid therapy with reversal of the symptoms of Cushing's syndrome, reduced weight and BMI, besides the signs and symptoms related to lupus erythematous systemic. It is suggested to continue the investigation, considering a larger number of patients, so that evidence of the reproducibility of the procedures adopted and the establishment of a clinical protocol secure.
\end{abstract}

Descriptors: Acupuncture; Lupus Erythematosus, Systemic; Auriculotherapy; Adrenal Cortex Hormones.

\section{Resumen}

El Lupus Eritematoso Sistémico (LES) es una enfermedad autoinmune de origen multifactorial caracterizada por la producción de auto anticuerpos patógenos. Según la Medicina Tradicional China (MTC) las enfermedades pueden ser provocadas por una desarmonía energética del organismo, considerando la salud como resultado del equilibrio de energía $(Q i)$, el cual puede ser alcanzado por medio de la acupuntura. En este artículo se reporta el caso de una paciente de 19 años que fue sometida a 26 sesiones de tratamiento con acupuntura por medio de auriculoterapia y asociación sistémica, buscando la disminución de los signos y síntomas relacionados al LES. Se observó una reducción de alrededor de 75\% en las áreas de dolor y de la ansiedad, así como la disminución de la dosis de corticoesteroides e Índice de Masa Corporal (IMC). Por lo tanto, la acupuntura demostró ser eficaz como tratamiento para el dolor, en la disminución de la ansiedad y como opción no farmacológica para la terapia con corticoides, la reversión de los síntomas del síndrome de Cushing, reducción del peso y el IMC, además de los signos y síntomas relacionados con el Lupus Eritematoso Sistémico. Se sugiere la continuación de la investigación, considerando un número mayor de pacientes, para que se evidencie la reproducibilidad de los procedimientos adoptados y se establezca un protocolo clínico seguro.

Descriptores: Acupuntura; Lupus Eritematoso Sistémico; Auriculoterapia; Corticoesteroides. 


\section{INTRODUÇÃO}

O Lúpus Eritematoso Sistêmico (LES) é uma doença autoimune caracterizada pela perda de tolerância aos antígenos próprios, produção de auto-anticorpos patogênicos e danos a múltiplos órgãos ${ }^{1}$. Sua etiologia não está totalmente elucidada, porém, é sabido que ela pode ser desencadeada por fatores genéticos, ambientais e hormonais que influenciam no descontrole do sistema imunológico ${ }^{2}$. Segundo a Medicina Tradicional Chinesa (MTC), um estado desarmônico pode levar ao desequilíbrio homeostático do organismo originar uma causa patológica e, quando a energia vital não pode vencer a energia nociva, a patologia se instala e se manifesta ${ }^{3}$.

Segundo dados do Ministério da Saúde a incidência do LES é de aproximadamente 1 a 22 casos a cada 100.000 pessoas por ano e a prevalência varia de 7 a 160 casos para cada 100.000 pessoas no mundo ${ }^{4}$. A sociedade Brasileira de Reumatologia estima que no Brasil cerca de 65.000 pessoas possuem lúpus sendo que a maioria são mulheres, cerca de 1 a cada 1.700 mulheres brasileiras possuem essa doença ${ }^{5}$.

A acupuntura é uma técnica de intervenção terapêutica baseada nos preceitos da MTC que considera a saúde o resultado do equilíbrio da energia $(Q i)$, dividida em polaridades (Yin/Yang), que o compõem e sustentam a vida ${ }^{6}$. Consiste na estimulação de pontos meridionais do organismo que representam os órgãos, essa estimulação é responsável por uma reação em cadeia que ativa neurônios cerebrais liberando substâncias que agirão no órgão correspondente ${ }^{7}$. Diferentemente da medicina ocidental convencional, considera o ser humano como um ser integral, sem barreiras entre mente, corpo e espírito ${ }^{8}$.

A auriculoterapia é uma das técnicas que compõe as práticas realizadas pela MTC, a qual consiste na estimulação de pontos específicos no pavilhão auricular para promover um balanço energético, criando influências positivas no paciente ${ }^{9}$. Essa técnica se encontra disponível no Sistema Único de Saúde (SUS) através da Portaria No 971 de 2006 que aprova a Política Nacional das Práticas Integrativas e Complementares ${ }^{10}$.

Diante disso, objetivou-se com esse estudo, avaliar as influências da acupuntura e da auriculoterapia no tratamento integrativo de um paciente com LES.

\section{CASO CLÍNICO}

Paciente do gênero feminino, 19 anos, $1,69 \mathrm{~m}$ de altura, pesando $75 \mathrm{Kg}$, características de corticodependência e diagnóstico de LES. Em fevereiro de 2015 procurou o Projeto de Extensão Permanente "Auriculoterapia Acadêmica" aprovado pelo Comitê de Ética e Pesquisa em Seres Humanos (CEPCEULJI/ULBRA) pelo parecer $\mathrm{n}^{\circ} 746.588 \mathrm{em} 11 \mathrm{de}$ Agosto de 2014, com a queixa principal de LES.

Durante a anamnese no tempo zero, antes do início do tratamento, a paciente declarou sentir dores nas articulações e principalmente nos joelhos, mãos, pés, além de fraqueza nos membros. Observou-se também que a paciente apresentava os efeitos colaterais do uso prolongado do corticoide. Relatou que fazia o uso de 30 $\mathrm{mg} / \mathrm{dia}$ de prednisona, azatioprina $(150 \mathrm{mg} / \mathrm{dia})$, Hidroxicloroquina $(400 \mathrm{mg} / \mathrm{dia})$, cálcio $(600 \mathrm{mg} / \mathrm{dia})$, vitamina D e Dipirona $(500 \mathrm{mg})$ em casos de dores intensas.

O tratamento convencional para LES, segundo o ministério da saúde envolve a utilização principalmente do antimaláricos: Cloroquina e Hidroxicloroquina e de glicocorticoides. Podendo também ser utilizados Azatioprina, Ciclofosfamida, Ciclosporina, Danazol, Metotrexato e Talidomida, escolhidos de acordo com o protocolo estabelecido pelo médico ${ }^{4}$.

A técnica complementar empregada foi a da aurículocibernética ${ }^{11}$ que consiste no estímulo primário de três pontos específicos: Shenmen, Rim e Simpático, para posterior uso de pontos auxiliares com base em literatura. As autodeclarações foram coletadas durante a anamnese, anterior ao tratamento (tempo zero), procedendo-se o mesmo a cada nova sessão em relação à sessão anterior. Os dados foram coletados entre fevereiro a novembro de 2015 , totalizando 26 sessões.

Foram utilizados os pontos: Cibernética (Shenmen, Rim e Simpático); imunidade, fígado; articulação do joelho; analgesia; ansiedade 1 e 2; pé-calcâneo, de acordo com as queixas apresentadas. $\mathrm{Na} 2^{\mathrm{a}}$ sessão a paciente relatou não ter feito o uso de analgésico, apresentou manchas na pele, o que incluiu o ponto alergia no protocolo de atendimento e iniciou o uso do medicamento Belimumab. A partir da $4^{\circ}$ sessão se iniciou a diminuição do corticoide.

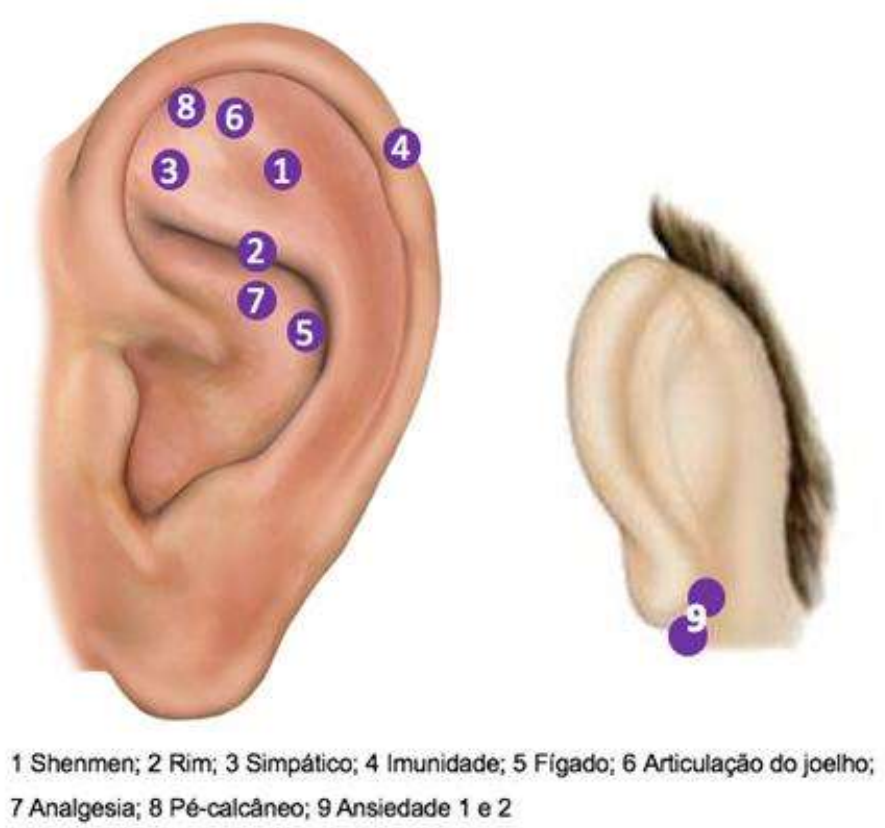

Figura 1. Pontos utilizados na $1^{\text {a }}$ Sessão, de acordo com Souza ${ }^{11}$ Fonte: Google imagens adaptado pelo autor

$\mathrm{Na} 7^{\mathrm{a}}$ sessão iniciou-se a intervenção com acupuntura sistêmica, foram utilizados os acupontos E36, R3, R7, BP6 e F3, baseados na pulsologia da 
paciente. A dose do corticoide era de $20 \mathrm{mg} / \mathrm{dia}$. A paciente relatou que não mudou seus hábitos alimentares, que praticava pouca de exercícios físicos devido as dores e mencionou também presença de sangue nas fezes. Na semana seguinte, a paciente declarou que voltou a menstruar e que o seu ciclo se normalizou e também não notou a presença de sangue nas fezes.

$\mathrm{Na} \quad 10^{\mathrm{a}}$ sessão observou-se através da pulsologia que o equilíbrio foi alcançado e a escolha do protocolo sistêmico passou a ser com a observação do pulso e queixas apresentadas pela paciente, a dose do corticoide foi diminuída para $17,5 \mathrm{mg} /$ dia e o peso era de $73,5 \mathrm{~kg}$. Na $13^{\mathrm{a}}$ sessão a paciente relatou a troca do medicamento Azatioprina pelo Metotrexato com a posologia de $12,5 \mathrm{mg}$ uma vez por semana e uma ingestão de $15 \mathrm{mg} /$ dia do corticoide. $\mathrm{Na} 17^{\mathrm{a}}$ sessão a paciente mencionou uma forte dor nas costas devido a uma fratura na vertebra L4 o que levou a utilização de pontos na aurícula para evitar dor no local da fratura.

$\mathrm{Na}$ última sessão $\left(26^{\mathrm{a}}\right)$ de tratamento a paciente relatou o uso de $8 \mathrm{mg} /$ dia do corticoide, não ter feio o uso de analgésico na última semana e seu peso era de $66 \mathrm{Kg}$.

De acordo com os dados obtidos durante as sessões, notou-se que o teste de Análise de Variância (ANOVA-1 way) revelou existir uma diferença altamente significativa para a distribuição dos valores $(\mathrm{p}<0,0001)$ para as variáveis ansiedade e dor, representadas pela Figura 2, onde ambas reduziram em $75 \%$ no final do tratamento.

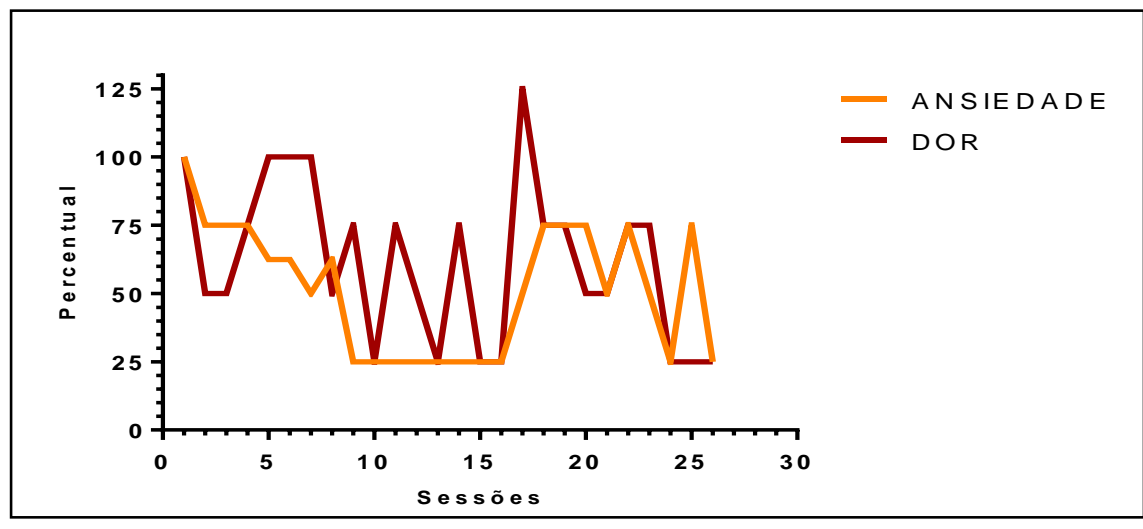

Figura 2. Distribuição das frequências relativas obtidas para os domínios dor e ansiedade em função das sessões

A relação entre a dose diária de corticoide e o Índice de Massa Corporal (IMC) se encontra representada na Figura 3, onde os testes estatísticos evidenciaram uma redução de $73,3 \%$ da dose do corticoide e $12 \%$ do IMC e a Análise de Variância revelou uma diferença altamente significativa $(\mathrm{p}<0,0001)$ para ambas as variáveis. A correlação de Pearson também evidenciou que a redução de ambos os domínios foi diretamente proporcional.

Durante o tratamento proposto também foram monitorados, a critério médico, os exames laboratoriais da paciente, onde foram analisados principalmente o número de leucócitos totais e linfócitos, os quais são influenciados pelo tratamento medicamentoso convencional.

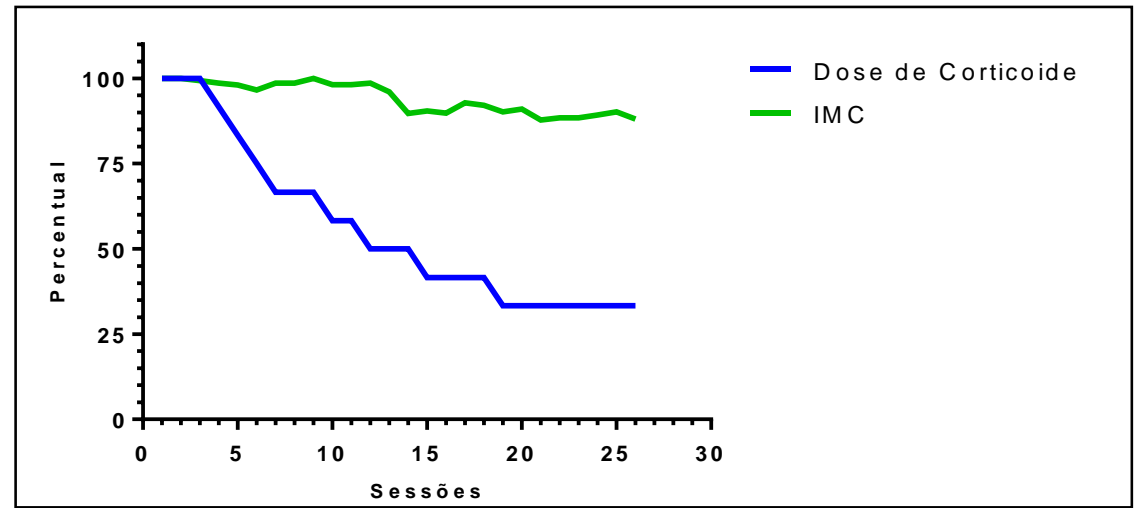

Figura 3. Distribuição das frequências relativas obtidas para os domínios dose de Corticoide e Índice de Massa Corporal (IMC) em função das sessões

A distribuição dos valores de leucócitos e linfócitos se encontra representada na Tabela 1 , onde foram comparados os períodos antes e após o tratamento.

Tabela 1. Distribuição dos valores de leucócitos e linfócitos por período de tempo

\begin{tabular}{lcccccccc}
\hline $\begin{array}{l}\text { Exame/ } \\
\text { Sessão }\end{array}$ & T0 & $\mathbf{1 0 ^ { \circ }}$ & $\mathbf{1 5 ^ { \circ }}$ & $\mathbf{2 0 ^ { \circ }}$ & $\mathbf{2 5 ^ { \circ }}$ & AT & VR & $\begin{array}{c}\text { Valor } \\
\text { de } \mathbf{p}\end{array}$ \\
\hline $\begin{array}{l}\text { Leucócitos } \\
\text { Totais }\end{array}$ & 5000 & 3100 & 2800 & 2000 & 4800 & 3500 & $\begin{array}{c}4500 \\
\mathrm{a} \\
10200\end{array}$ & $\begin{array}{c}\mathrm{p}<0,001 \\
* * *\end{array}$ \\
\hline Linfócitos & 1100 & 899 & 1008 & 820 & 864 & 840 & $\begin{array}{c}1150 \\
\mathrm{a} \\
4590\end{array}$ \\
\hline *T0 = tempo zero, AT = após o tratamento, VR= Valor Referencial. Todos os valores são em
\end{tabular}

milímetros cúbicos. **Teste do qui-quadrado

De acordo com o teste $\mathrm{t}$ pareado não houve diferença significativa entre os parâmetros avaliados $(\mathrm{p}=0,29)$, a análise da variância não identificou uma representatividade estatística $(\mathrm{p}<0,05)$, assim como, o teste de múltiplas análises de Dunnett que comparou os valores de cada sessão com a média dos parâmetros do intervalo de referência. Porém o teste do qui-quadro revelou uma associação positiva entre os valores de leucócitos totais e o número de linfócitos.

\section{DISCUSSÃO}

A acupuntura é um instrumento terapêutico que considera um protocolo clínico para o restabelecimento integral do paciente, buscando o equilíbrio energético e permitindo que sejam tratados diferentes sinais e sintomas em um mesmo momento, procurando a melhoria da qualidade de vida ou reestabelecer a saúde do paciente $^{7,12}$.

Recentemente um estudo retrospectivo analisou dados de aproximados 23 mil pacientes por 10 anos e concluiu sobre os benefícios da MTC como terapia combinada na sobrevivência de pacientes com $\operatorname{LES}^{13}$. A efetividade da acupuntura no manejo dos sintomas do LES foi relatada por Mooventhan e Nivethitha ${ }^{14}$ que registraram a diminuição da dor e da ansiedade, sendo 
estas diretamente relacionadas com a saúde e qualidade de vida desses pacientes.

A diminuição da dor e da ansiedade também foi observada neste estudo, bem como uma oscilação entre elas. Provavelmente esse quadro está associado ao estresse gerado pela ansiedade, formando um ciclo vicioso no qual a ansiedade gera estresse, provocando uma redução na tolerância à dor e elevando o nível de ansiedade $^{15}$. Tal evidência pode ser sustentada na conclusão de Silva e Ribeiro Filho ${ }^{16}$ que tratam a dor como sendo algo modificável pela memória, expectativas e emoções, podendo ser originada de estímulos sensoriais ou lesões neurológicas.

$\mathrm{O}$ presente relato de caso sugere que a aurículoterapia e associada com acupuntura sistêmica foi efetiva para o alivio da dor revelando uma redução de $75 \%$ da queixa após a intervenção, tal como observaram Greco et $\mathrm{al}^{17}$ em seu estudo piloto onde recomendam a acupuntura como um método não-farmacológico para controlar a dor em pacientes com lúpus. Assim como sugere também a sua efetividade para diminuição da ansiedade, também reduzida em $75 \%$, corroborando com Shayestehfar et $\mathrm{al}^{18}$ que verificaram que a acupuntura foi eficaz para diminuir a ansiedade através da utilização de marcadores fisiológicos e psicológicos em 45 atletas adolescentes antes da competição.

Também se observou picos que caracterizam um rápido aumento na intensidade da dor. Tal fato esteve associado a uma fratura por compressão na vertebra L4, o que pode acontecer em aproximadamente 30 a $35 \%$ dos pacientes com Síndrome de Cushing, devido as doses elevadas do corticoide e ao uso crônico que podem provocar osteoporose secundária, efeito colateral grave relacionado glicocorticoides ${ }^{19,20}$.

O Protocolo Clínico e Diretrizes Terapêuticas do Ministério da Saúde estabelece os esquemas usuais de prednisona para pacientes com LES, podendo ser administradas uma dose baixa de 7,5 $\mathrm{mg} /$ dia até uma dose muito alta de $100 \mathrm{mg} / \mathrm{dia}$ e assim que possível devem ser reduzidos gradualmente até a suspensão ${ }^{4}$. Durante o período de tratamento foi observada uma redução gradativa do corticoide, ocorrendo uma relação entre a diminuição da dose e a redução do peso e consequentemente do Índice de Massa Corporal (IMC), sendo que a dosagem do corticoide reduziu em 73,3\% e o IMC em $12 \%$.

De acordo com Santos et $\mathrm{al}^{21}$ o principal distúrbio nutricional de paciente com LES é o excesso de peso. $53,3 \%$ dos pacientes avaliados por Salicio et $\mathrm{al}^{22}$ apresentaram sobrepeso, assim como a paciente do presente estudo. Porém, após as práticas da MTC a paciente passou a ocupar a faixa de peso normal. Vale ressaltar que segundo a mesma, não houve mudança nos hábitos alimentares ou na prática de exercícios físicos regulares, o que comprova a efetividade do tratamento na redução do seu IMC.
Diversos estudos relatam a ação da auriculoterapia e da acupuntura para a redução de peso, como o estudo piloto de Ito et $\mathrm{al}^{23}$ que evidencia diferenças significativas na redução do peso e na supressão de grelina, reduzindo o apetite e também como no estudo de $\operatorname{Haddad}^{24}$ onde o peso e o IMC de trabalhadores de um hospital universitário foram reduzidos, bem como o seu apetite controlado após a intervenção.

Com relação aos exames laboratoriais, diferentemente dos resultados apresentados por Ourique et $\mathrm{al}^{25}$, que evidenciaram um resultado regulador na imunidade, no presente estudo verificou-se baixa imunidade, podendo ser observada na tabela 1 , onde os leucócitos só se mantiveram dentro do valor referencial uma vez durante o período de tratamento. O que pode ser explicado pela doença ser muito influenciada pelo estado emocional e pelo uso de glicocorticoides que vem sendo associado aumento do risco de infecções bacterianas causadas por microrganismos gram-negativos, micobactérias, criptococos, listeria e nocardia ${ }^{26}$.

Estudos demostram que existe um padrão diferente de resposta imune nos pacientes acometidos com LES quando comparados com pessoas saudáveis, podendo estar ligados a piora da doença ${ }^{27}$, onde as oscilações desse estado, podem também levar a oscilações no quadro clínico e nas queixas apresentadas pelo paciente. A associação evidenciada entre o número de leucócitos totais e linfócitos demonstra que quando ocorre uma variação em uma das variáveis a outra também sofre mudanças, ocasionando leucopenia e linfopenia, que juntamente com a elevação da taxa de sedimentação dos eritrócitos e o anti-DNA anticorpo nativo, evidenciadas no presente caso, têm sido associadas à atividade do $\mathrm{LES}^{28}$.

Além disso, as técnicas da MTC também se mostraram efetivas na diminuição da quantidade de medicamentos ingeridos pela paciente, sendo evitada a administração de dipirona, cujo uso tem sido relacionado a casos de agranulocitoses desde 1935, gerando inúmeros estudos que provocaram a suspensão da administração em diversos países da Europa e Estados Unidos, entre outros ${ }^{29}$.

Outro benefício das técnicas da MTC observado durante o estudo foi o retorno no ciclo menstrual e de sua regularidade, o qual se encontra alterado em mulheres que apresentam a doença como observado no estudo de Ulug et $\mathrm{al}^{30}$, ou seja, mulheres acometidas com LES apresentaram maior irregularidade menstrual e reservas ovarianas mais baixas comparadas com as que não possuem essa patologia.

Assim os resultados apresentados neste estudo também corroboram com o relato de caso de Donoyama et $\mathrm{al}^{31}$ onde a acupuntura pode ter influenciado positivamente para o retorno do ciclo menstrual em uma atleta. 


\section{CONCLUSÃO}

A Acupuntura, por meio da auriculoterapia e da associação sistêmica, foi efetiva na redução da dor, diminuição da ansiedade e da corticoterapia, com inversão dos sintomas da síndrome de Cushing. Influenciou também na redução do peso e do IMC, além de diminuir os sinais e sintomas relacionados ao Lúpus Eritematosos Sistêmico. Todavia, novos estudos devem ser realizados, considerando um número maior de pacientes para que um delineamento distinto evidencie a reprodutibilidade dos procedimentos e se estabeleça um protocolo clínico seguro.

\section{REFERÊNCIAS}

1. Liu Z, Davidson A. Taming lupus-a new understanding of pathogenesis is lea-ding to clinical advances. Nature Medicine. 2006; 18:871-882

2. Lopes ACQ. Lúpus eritematoso: principais sintomas, diagnóstico e terapêutica. Departamento Nacional de Pós-Graduação e Atualização. Campinas, 2013. Disponível em: http:// www.redentor.inf.br/arquivos/pós/publicações/2005 2013Ana\%20Carolinapdf

3. Machado E, Simabukuro M, Reinato SG. O uso do stiper no tratamento da osteartrose de joelho na medicina tradicional chinesa. Centro de Estudo de Acupuntura e Terapia Alternativa. 2014. 64p.

4. Brasil. Ministério da Saúde. Secretaria de Atenção a Saúde. Portaria No 100 de 7 de fevereiro de 2013. Protocolo Clínico e Diretrizes Terapêuticas do Lúpus Eritematoso Sistêmico.

5. Sociedade Brasileira de Reumatologia. Lúpus. São Paulo: Sociedade Brasileira de Reumatologia; 2011.

6. Yamamura Y. Acupuntura Tradicional: A Arte de Inserir. São Paulo: Roca; 2001.

7. Serpa LS, Watanabe LAR, Carvalho, AL. Utilização da acupuntura em órgãos e vísceras, através dos pontos Bei Shu: revisão bibliográfica. Amazônia Sci \& Health. 2016; 4(1): 35-40.

8. Kurebayash LFS, Gnatta JR, Borges TP, Belisse G, Coca S, Minami A, et al. Aplicabilidade da auriculoterapia com agulhas ou sementes para diminuição de estresse em profissionais de enfermagem. Rev Esc Enferm USP. 2012; 46(1):8995.

9. León ALC, Alberro YD, Fernández DS, González JOC. Tratamiento de hábitos bucales deformantes con técnica de auriculoterapia. Rev Ciencias Médicas, Pinar Del Río. 2014; 18(6):1027-35.

10. Brasil. Ministério da Saúde. Gabinete do Ministro. Portaria $\mathrm{n}^{\circ}$ 971, de 03 de maio de 2006. Política Nacional de Práticas Integrativas e Complementares (PNPIC) no Sistema Único de Saúde.

11. Souza, MP. Tratado de Auriculoterapia. Brasília: Artes Gráficas; 2013.
12. Nascimento TAA, Olcerenko DR. A acupuntura como ferramenta da assistência de enfermagem. Rev Enferm UNISA. 2009; 10(2):178-81.

13. Ma YC, Lin CC, Li CI, Chiang JH, Li TC, Lin JG. Traditional Chinese medicine therapy improves the survival of systemic lupus erythematosus patients. Semin Arthritis Rheum. 2016; 45(5):596-603.

14. Mooventhan A, Nivethitha L. Effects of acupuncture and massage on pain, quality of sleep and health related quality of life in patient with systemic lupus erythematosus. J Ayurveda Integr Med. 2014; 5(3):186-9.

15. Siqueira AMP, Oliveira PC, Shcaira VRL, Ambrosano GMB, Ranali J, Volpato MC. Anxiety and pain associated with local anesthesia and periodontal therapy. Rev Odontol UNESP. 2006; 35(2):171-4.

16. Silva JA, Ribeiro Filho, NP. Pain as a psychophysical problem. Rev Dor. 2011; 12(2):138-51.

17. Greco CM, Kao AH, Maksimowicz-McKinnon K, Glick RM, Houze M, Sereika SM, et al. Acupuncture for systemic lupus erythematosus: a pilot RCT feasibility and safety study. Lupus. 2008; 17(12):1108-16.

18. Shayestehfar M, Seif-Barghi T, Zarei S, Mehran A. Acupuncture Anxiolytic Effects on Physiological and Psychological Assessments for a Clinical Trial. Scientifica (Cairo). 2016;2016:4016952. doi: $10.1155 / 2016 / 4016952$

19. Magalhães MS, Silva FV. Glicocorticoides e o desenvolvimento da osteoporose em pacientes com síndrome de cushing: uma revisão. Interfaces Científicas. 2012; 1(1):41-52.

20. Frankel B, White W, Tuckermann J. Glucocorticoid-Induced Osteoporosis. Adv Exp Med Biol. 2015; 872:179-215.

21. Santos FMM, Borges MC, Correia MITD, Telles RW, Lanna CCD. Avaliação do estado nutricional e da atividade física em pacientes com lúpus eritematoso sistêmico. Rev Bras Reumatol. 2010; 50(6):631-45.

22. Salicio VAMM, Leite CA, Arruda LKA, Santin $\mathrm{ACW}$, Matos MBP, Galera MF et al. Avaliação da qualidade de vida em pacientes com Lúpus Eritematoso Sistêmico, atendidos no hospital Universitário em Mato Grosso - Brasil. Rev Bras Ciênc Saúde. 2013;11(36):50-6.

23. Ito H, Yamada S, Kira Y, Tanaka T, Matsuoka R. The effects of auricular acupuncture on weight reduction and feeding-related cytokines: a pilot study. BMJ Open Gastroenterol. 2015 Feb 9;2(1):e000013. doi: 10.1136/bmjgast-2014-000013

24. Haddad ML, Marcon SS. Acupuntura e apetite de trabalhadores obesos de um hospital universitário. 
Acta Paul Enferm. 2011; 24(5):676-82.

25. Ourique AAB, Oliveira SG, Meneghetti A. Ação da acupuntura no aumento da imunidade no paciente com lúpus eritematoso sistêmico. Ciência, Cuidado e Saúde UEM. 2008; 7(Suppl 2) doi.org/10.4025/cienccuidsaude.v7i0

26. Skare TL, Dagostini JS, Zanardi PI, Nisihara RM. Infecções e lúpus eritematoso sistêmico. Einstein (São Paulo). 2016; 14(1):47-51.

27. Nery FG, Borba EP, Neto FL. Influência do estresse psicossocial no Lúpus Eritematoso Sistêmico. Rev Bras Reumatol. 2004; 44(5):355-61.

28. Cassano G, Roverano S, Paira S, Moral RC, Barrionuevo A, Rillo $\mathrm{O}$ et al. Eritrosedimentación, leucopenia, linfopenia y anticuerpo anti-DNA nativo en lupus eritematoso sistémico. Asociación con actividad y daño orgânico. Rev argent reumatol. 2008; 19(1):14-21.

29. Lucchetti G, Granero AL, Almeida LGC, Battistella VM. Pancitopenia associada ao uso de dipirona. Relato de caso. Rev Soc Bras Clín Méd. 2010; $8(1): 72-6$.

30. Ulug P, Oner G, Kasap B, Akbas EM, Ozcicek F. Evaluation of ovarian reserve tests in women with systemic lupus erythematosus. Am J Reprod Immunol. 2014; 72(1):85-8

31. Donoyama N, Hotoge S, Ohkoshi N. Acupuncture might have contributed to improving amenorrhoea in a top athlete. Acupunct Med. 2011; 29(4):304-6.

\section{CONFLITO DE INTERESSES}

Os autores declaram não haver conflitos de interesse.

\section{AUTOR PARA CORRESPONDÊNCIA}

\section{Magda Fardim Dalcin}

magfardindalcin@gmail.com

Submetido em 20/08/2016 Aceito em 13/10/2016 